\title{
Updates from FragMAX, a crystallographic fragment screening platform at MAX IV Laboratory Tobias Krojer
}

MAX IV Laboratory, Lund University, PO Box 118, S-221 00 Lund, Sweden

tobias.krojer@maxiv.lu.se

Fragment-based lead discovery (FBLD) is by now an established drug development strategy, which has so far delivered four novel drugs and more than 40 additional molecules in clinical trials. Starting points for FBLD are usually found by biophysical screening of fragment libraries with several hundred and up to a few thousand compounds. Crystal-based fragment screening has become increasingly popular over the last few years, facilitated by automation, improvements in beamline instrumentation and software development. The FragMAX facility provides a new user platform for crystallographic fragment screening at BioMAX, the first operational beamline for macromolecular crystallography at MAX IV Laboratory. This presentation will provide an overview of the different components of the FragMAX facility and describe the screening process. It will highlight different modes of access and outline planned developments.

The FragMAX platform started serving external users in 2019 and has since established an international user program that is open to academic and industrial research groups. The platform consists of three main components: (i) a crystal preparation facility, (ii) diffraction data collection at BioMAX and (iii) FragMAXapp, an intuitive web-based tool for large-scale data processing. The facility provides free access to several fragment libraries, notably, the in-house developed FragMAXlib. The screening processes have been adjusted throughout the COVID-19 pandemic and our aim is to further develop the platform so that users with different levels of experience can routinely achieve actionable screening hits for their targets. While the facility was conceptualized for crystal-based fragment screening, its design is entirely generic. It can therefore be used for other applications that require large-scale crystal preparation and potential new applications will be discussed at the end of the presentation.

Keywords: fragment screening, protein crystallography, drug discovery 